\title{
SOME CRITERIA FOR THE NONEXISTENCE OF CERTAIN FINITE LINEAR GROUPS
}

\author{
HARVEY I. BLAU
}

\begin{abstract}
Let $p$ be a prime and $G$ a finite group, not of type $L_{2}(p)$, with a cyclic Sylow $p$-subgroup $P$. Assume that $G=G^{\prime}$. The purpose of this note is to put some rather stringent lower bounds on the degree $d$ of a faithful indecomposable representation of $G$ over a field of characteristic $p$ given certain conditions on the normalizer $N$ and the centralizer $C$ of $P$ in $G$. In particular, if the center of $G$ has order 2 and $|N: C|=p-1$, then $d \geqq p-1$.
\end{abstract}

This paper began when the author realized that some of the methods in [1] were more powerful than he originally believed. Consequently, elements of the argument below appeared in slightly less general form (and with weaker application) in [1, §5]. Although the blanket hypotheses of that section do not exactly coincide with those of our assertions (we do not assume here that $p \geqq 13$ or that $G$ is not of type $L_{2}(p)$ in Lemma 1 below), it should be clear that the results we quote from $[1, \S 5]$ are indeed valid in the context where they are applied.

Throughout the paper $G$ denotes a finite group, $p$ an odd prime, $P$ a Sylow $p$-subgroup of $G . N$ and $C$ are, respectively, the normalizer and centralizer of $P$ in $G, e=|N: C|, t=(p-1) / e$, and $z$ is the order of $Z$, the center of $G . K$ is a field of characteristic $p$ which is a splitting field for all subgroups of $G$. If $M$ is a $K G$-module, $M^{*}$ denotes its dual. The linear character $\alpha: N / P \rightarrow K$ is as defined in [1]. $v_{2}$ is the usual 2-adic valuation on the rationals.

Hypothesis A. $|P|=p$ and $N / P$ is abelian.

Hypothesis B. $P$ is cyclic, $G$ is not of type $L_{2}(p)$, and there is a faithful indecomposable $K G$-module $L$ of dimension $d=p-s \leqq p$.

Hypothesis B implies Hypothesis A by [2]. If group $G$ and module $L$ satisfy Hypothesis B, then so do $J$ and $L_{J}$, where $J$ is the intersection of the derived series of $G$. So the assumption $G=G^{\prime}$ is not a severe restriction.

Presented to the Society, January 16, 1974; received by the editors August 13, 1973. AMS (MOS) subject classifications (1970). Primary 20C20, 20C05; Secondary $20 \mathrm{D} 05$.

Key words and phrases. Indecomposable modular representation, small degree, cyclic Sylow $p$-subgroup.

(c) American Mathematical Society 1974 
Our first result generalizes [1, Lemma 5.15], and has almost the same proof.

Lemma 1. Assume Hypothesis A and that $G=G^{\prime}$. Suppose there exist indecomposable KG-modules $L$ and $M$ of dimension $d$ with $p / 2<d<p$ such that $M \approx L^{*}$ but the nonprojective summands of $L \otimes M$ are self-dual. Then

$$
\begin{array}{ll}
d \geqq p-t & \text { ( } t \text { odd }), \\
\geqq p-t+1 & \text { (t even }) .
\end{array}
$$

Proof. Let $L_{N}=V_{d}(\lambda), M_{N}=V_{d}(\gamma)$ as in [1, §4]. Let $s=p-d$. $M \approx L^{*}$ implies $\lambda \gamma \alpha^{s} \neq 1$ [1, Lemma 2.3]. As in [1, §5], the nonprojective summands of $L \otimes M$ are $L_{i}, 0 \leqq i \leqq s-1$, where $\operatorname{dim} L_{i}=2 i+1+m_{i} p$ and

$$
L_{i_{N}}=V_{2 i+1}\left(\lambda \gamma \alpha^{s+i}\right)+\sum_{j \in \mathscr{S}_{i}} V_{p}\left(\lambda \gamma \alpha^{s+j}\right) .
$$

As remarked in $[1, \S 5]$, the $L_{i}$ are self-dual if and only if $\left(\lambda \gamma \alpha^{s}\right)^{2}=1$.

Suppose $m_{i}$ is odd. Then, as in the proof of [1, Lemma 5.5], there is an odd number of $j \equiv 0(\bmod e)$ in $\mathscr{S}_{i}$ if $t$ is even, and an odd number of $j \equiv e / 2(\bmod e)$ in $\mathscr{S}_{i}$ if $t$ is odd. Suppose $m_{i}$ is even. Then $2 i+1+m_{i} p$ is odd, so $[1,(5.6)]$ and $\left(\lambda \gamma \alpha^{s}\right)^{2}=1$ imply

$$
1=\lambda \gamma \alpha^{s} \prod_{j \in \mathscr{S}_{i} ; 2, j \equiv 0(\bmod e)} \alpha^{j} .
$$

Since $\lambda \gamma \alpha^{s} \neq 1$, there must exist $j \in \mathscr{S}_{i}$ with $j \not \equiv 0(\bmod e), 2 j \equiv 0(\bmod e)$. Hence $e$ is even and $j \equiv e / 2(\bmod e)$. Furthermore, there must be an odd number of such $j$ in $\mathscr{S}_{i}$. Since $m_{i}$ is even, there must also be $j \in \mathscr{S}_{i}$ with $j \equiv 0(\bmod e)$. So each $\mathscr{S}_{i}$ contains some $j \equiv 0(\bmod e)$ if $t$ is even, or $j \equiv e / 2(\bmod e)$ if $t$ is odd. Since $|\langle\alpha\rangle|=e,[1,(5.2)]$ implies $s \leqq t$ if $t$ is odd, and $s \leqq t-1$ if $t$ is even.

Theorem 2. Assume Hypothesis B and $G=G^{\prime}$. Suppose there is a positive integer $n$ such that ze $\mid 2\left(p^{n}-1\right)$ but ze $\nmid p^{n}-1$. Then

$$
\begin{array}{ll}
d \geqq p-t & (t \text { odd }), \\
\geqq p-t+1 & (t \text { even }) .
\end{array}
$$

Proof. Let $q=p^{n}$, and $\sigma$ be the isomorphism of $K$ into $K$ given by $x^{\sigma}=x^{q}$, all $x \in K$. Let $\mathscr{L}$ be a representation of $G$ with underlying module $L$, and for each $g \in G$, let $\mathscr{L}(g)^{\sigma}$ be the matrix obtained by replacing each entry $a_{i j}$ of $\mathscr{L}(g)$ by $a_{i j}^{\sigma}$. Then $g \rightarrow \mathscr{L}(g)^{\sigma}$ defines a representation of $G$ with an underlying indecomposable $K G$-module of dimension $d$ which we will call $L^{\sigma}$. If $L_{N}=V_{d}(\lambda)$, then $\left(L^{\sigma}\right)_{N}=V_{d}\left(\lambda^{\sigma}\right)$, where $\lambda^{\sigma}=\lambda^{q}$. $\left(L^{*}\right)_{N}=V_{d}\left(\lambda^{-1} \alpha^{-s}\right)\left[1\right.$, Lemma 2.3]. Note that $\lambda^{\sigma}\left(\lambda^{-1} \alpha^{-s}\right) \alpha^{s}=\lambda^{q-1}$. 
Since $z e=|N / P|[2]$ and $\lambda$ is a linear character: $N / P \rightarrow K, z e \mid 2(q-1)$ implies $\lambda^{2(q-1)}=1$. It follows that the nonprojective summands of $L^{\sigma} \otimes L^{*}$ are self-dual. The conditions $z e \mid 2(q-1), z e \nmid q-1$ imply

$$
v_{2}(z)+v_{2}(e)=1+v_{2}(q-1) .
$$

Since $e \mid p-1$, it follows that $z$ is even and hence $d$ is even by [1, Proposition 5.1].

Suppose $\lambda^{q-1}=1$. Since $\lambda$ is faithful on cyclic $Z$ [1, Proposition 5.1], $z \mid q-1$. If $e$ is odd, we have $v_{2}(z)=1+v_{2}(q-1)$, a contradiction, so $e$ is even. Then the first paragraph of the proof of [1, Theorem 5.12] shows that $\left(\lambda^{2}\right)^{2 i+1+m_{i} p}$ is an odd power of $\alpha$ for all $0 \leqq i \leqq s-1$ with $i \equiv(p+1) / 2(\bmod 2)$, where $2 i+1+m_{i} p$ is the dimension of the summand of $L \otimes L$ with Green correspondent $V_{2 i+1}\left(\lambda^{2} \alpha^{s+i}\right)$. Since we may assume $d<p-1$, there exist such $i$. Because $z \mid 2\left(2 i+1+m_{i} p\right)[1,(5.10)]$ and $\lambda^{z} \in\langle\alpha\rangle$, it follows that $\lambda^{z}$ is an odd power of $\alpha$. Now since $1=\left(\lambda^{z}\right)^{(\alpha-1) / z}$, we have $v_{2}((q-1) / z) \geqq v_{2}(e)$ which contradicts (3). Hence, $\lambda^{q-1} \neq 1$, so $L^{*} \approx\left(L^{\sigma}\right)^{*}$ and Lemma 1 may be applied.

Remarks. (i) [1, Theorem 5.18] is a special case of Theorem 2, with $z=2, t$ odd, and the (now unnecessary) restriction $L \approx L^{*}$.

(ii) The following numerical cases listed in $[1, \S 8]$ are eliminated by Theorem 2 (each 4-tuple is an instance of $p, d, z, e):(29,24,8,7)$, $(29,24,4,14),(29,26,26,28),(31,24,2,30)$.

(iii) The assumptions of Theorem 2 are satisfied if we have Hypothesis B, $G=G^{\prime}, t$ odd, and $z=2^{k}$, where either $p \equiv 1(\bmod 4)$ and $k$ is any positive integer, or $p \equiv 3(\bmod 4)$ and $k=1$ or $k>v_{2}(p+1)$. In particular, if $t=1$ and $z=2$, then $d \geqq p-1$. This bound is best possible, as there is a group $G=G^{\prime}$ satisfying Hypothesis B, with $p=7, z=2$, and $e=d=6$, such that $G / Z$ is the Hall-Janko group of order 604,800 [3]. Feit has shown, in work not yet published, that there is no $G=G^{\prime}$ satisfying Hypothesis B with $p=11, z=2$, and $e=d=10$. For $p>11$, the existence of relevant groups with $z=2$ and $e=d=p-1$ is apparently unknown.

Some notation is needed to state the next theorem. Assume Hypothesis B and $G=G^{\prime}$. Let $L_{N}=V_{d}(\lambda)$. Define the integer $x \quad(=x(L)$, unique modulo $e$ ) by $\lambda^{z}=\alpha^{[z(d-1) / 2]+x}$ where square brackets denote the greatest integer symbol. Since the determinant of the action of each element of $G$ on $L$ is 1 , [1, Lemma 2.3] implies $\lambda^{d}=\alpha^{d(d-1) / 2}$ Now $z \mid d$, so when $z$ is even, $\lambda^{d}=\left(\lambda^{z}\right)^{d / z}$ implies $x d / z \equiv 0(\bmod e)$.

THeOREM 4. Assume Hypothesis B with $G=G^{\prime}, t$ odd, and $d$ even. Suppose there is an odd positive integer $n$ such that $p^{n} \equiv \pm 1(\bmod z)$ and $x\left(p^{n} \mp 1\right) / z \equiv 0(\bmod e)$. Then $d \geqq p-t$. 
Proof. Let $q=p^{n}$, and let $\sigma$ be as in Theorem 2 (so $\left(L^{\sigma}\right)_{N}=V_{d}\left(\lambda^{q}\right)$ ). Since we may assume $d<p-1,[1$, Theorem 5.12] implies $z$ is even.

Suppose $q \equiv 1(\bmod z)$ and $x(q-1) / z \equiv 0(\bmod e)$. Then

$$
\begin{aligned}
\lambda^{q-1} & =\left(\lambda^{z}\right)^{(q-1) / z}=\alpha^{((q-1)(d-1) / 2)+(x(q-1) / z)} \\
& =\alpha^{((q-1) / 2)(d-1)}=\alpha^{((p-1) / 2)(\text { odd integer })}=\alpha^{e / 2}
\end{aligned}
$$

since $d$ is even and $n, t$ are odd. Thus $\lambda^{q-1} \neq 1,\left(\lambda^{q-1}\right)^{2}=1$. It follows that $L^{\sigma}$ and $L^{*}$ satisfy the hypotheses of Lemma 1 .

Suppose $q \equiv-1(\bmod z)$ and $x(q+1) / z \equiv 0(\bmod e)$. Then

$$
\lambda^{a+1}=\left(\lambda^{z}\right)^{(a+1) / z}=\alpha^{((a+1)(d-1) / 2)+(x(a+1) / z)}=\alpha^{((a-1)(d-1) / 2)+(d-1)}=\alpha^{(e / 2)-s} .
$$

So $\lambda^{a} \lambda \alpha^{s} \neq 1,\left(\lambda^{a} \lambda \alpha^{s}\right)^{2}=1$, whence $L^{\sigma}$ and $L$ satisfy the hypotheses of Lemma 1.

Corollary 5. Assume Hypothesis B with $G=G^{\prime}, t$ odd, and $d$ even. Suppose there is an odd positive integer $n$ such that $p^{n} \equiv \pm 1(\bmod d)$. Then $d \geqq p-t$.

MORe Remarks. (iv) Theorem 4 eliminates the cases $(17,14,14,16)$ and $(31,28,14,30)$ from $[1, \S 8]$.

(v) The numerical assumptions of Corollary 5 hold if $t$ is odd and $d=2 r, r$ a prime such that $r \equiv 3(\bmod 4)$.

\section{REFERENCES}

1. H. I. Blau, Under the degree of some finite linear groups, Trans. Amer. Math. Soc. 155 (1971), 95-113. MR 43 \#367.

2. W. Feit, Groups with a cyclic Sylow subgroup, Nagoya Math. J. 27 (1966), 571-584. MR 33 \#7404.

3. J. H. Lindsey II, On a six dimensional projective representation of the Hall-Janko group, Pacific J. Math. 35 (1970), 175-186. MR 42 \#7769.

Department of Mathematical Sciences, Northern Illinois University, DeKalb, ILLINOIS 60115 